\title{
Probe-based confocal laser endomicroscopy and fluorescence- based enhanced reality for real-time assessment of intestinal microcirculation in a porcine model of sigmoid ischemia
}

\author{
Michele Diana $\cdot$ Bernard Dallemagne $\cdot$ Hyunsoo Chung $\cdot$ Yoshihiro Nagao • \\ Peter Halvax • Vincent Agnus • Luc Soler • Veronique Lindner • Nicolas Demartines • \\ Pierre Diemunsch • Bernard Geny $\cdot$ Lee Swanström • Jacques Marescaux
}

Received: 16 December 2013/ Accepted: 6 May 2014/Published online: 17 June 2014

(C) Springer Science+Business Media New York 2014

\begin{abstract}
Background and aim Surgeons currently rely on visual clues to estimate the presence of sufficient vascularity for safe anastomosis. We aimed to assess the accuracy of endoluminal confocal laser endomicroscopy (CLE) and laparoscopic fluorescence-based enhanced reality (FLER), using near-infrared imaging and fluorescence from injected Indocyanine Green, to identify the transition from ischemic to vascular areas in a porcine model of mesenteric ischemia. Methods Six pigs underwent 1-h sigmoid segmental ischemia. The ischemic area was evaluated by clinical assessment and FLER to determine presumed viable margins. For each sigmoid colon, 5 regions of interest (ROIs) were identified: ischemic (ROI 1), presumed viable margins ROI $2 \mathrm{a}$ (distal) and 2b (proximal), and vascular areas 3a
\end{abstract}

M. Diana $(\bowtie) \cdot$ B. Dallemagne $\cdot$ H. Chung $\cdot$ Y. Nagao .

P. Halvax · V. Agnus $\cdot$ L. Soler $\cdot$ L. Swanström $\cdot$ J. Marescaux IRCAD Research Institute against Cancer of the Digestive System and IHU Minimally Invasive Image-Guided Surgical Institute, 1, place de 1'Hôpital, 67095 Strasbourg, France e-mail: michele.diana@ircad.fr

M. Diana $\cdot$ N. Demartines

Department of Visceral Surgery, CHUV, University Hospital of Lausanne, Lausanne, Switzerland

M. Diana · B. Geny

Institute of Physiology, EA 3072: Oxidative Stress,

Mitochondria and Muscle Protection, Strasbourg, France

V. Lindner

Department of Pathology, Hospital of Mulhouse, Mulhouse,

France

P. Diemunsch

Department of Anesthesiology, University of Strasbourg,

Strasbourg, France (distal) and 3b (proximal). After injection of fluorescein, CLE scanning of the mucosa from the ischemic area toward viable margins was performed. Capillary blood samples were obtained by puncturing the serosa at the ROIs, and capillary lactates were measured with the $\mathrm{EDGE}^{\circledR}$ analyzer. Results Capillary lactates were significantly higher at ROI 1 (4.91 mmol/L) when compared to resection margins (2.8 mmol/L; mean difference: $2.11 ; p<0.05)$ identified by FLER. There was no significant difference in lactates between ROI1 and resection margins identified by clinical evaluation. In $50 \%$ of cases, ROI $2 \mathrm{a}_{\text {CLINIC }}-2 \mathrm{~b}_{\text {CLINIC }}$ were considered to match $\left(<1 \mathrm{~cm}\right.$ distance) with ROI $2 \mathrm{a}_{\mathrm{FLER}}-$ $2 b_{\text {FLER }}$. Confocal analysis revealed specific clues to identify the transition from ischemic to viable areas corresponding to those assessed by FLER in 11/12 cases versus $7 / 12$ for those identified by clinical evaluation.

Conclusions In this experimental model, FLER and CLE were more accurate than clinical evaluation to delineate bowel vascularization.

Keywords Confocal laser endomicroscopy · Anastomotic perfusion - Fluorescence-based enhanced reality

$\begin{array}{ll}\text { Abbreviations } \\ \text { CLE } & \text { Confocal laser endomicroscopy } \\ \text { FLER } & \text { Fluorescence-based enhanced reality } \\ \text { ICG } & \text { Indocyanine green } \\ \text { IMA } & \text { Inferior mesenteric artery } \\ \text { IVM } & \text { Intravital microscopy } \\ \text { NIRS } & \text { Near-infrared spectroscopy } \\ \text { ROI } & \text { Regions of interest } \\ \text { SpO2 } & \text { Arterial oxygen saturation } \\ \text { StO2 } & \text { Hemoglobin oxygen saturation } \\ \text { VLS } & \text { Visible light spectroscopy }\end{array}$


In ablative surgery, adequate perfusion of segments to be anastomosed is a key factor for optimal healing and minimization of leakage and stenosis [1] risks.

Surgeons rely on subjective clinical features to estimate intestinal microcirculation and select the anastomotic site such as color of the serosal surface, vascular pulsations, and/or bleeding from marginal arteries. However, the accuracy of clinical evaluation to predict anastomotic complications, irrespective of the surgeon's experience, is low [2]. It is also unknown whether the mucosal surface of the bowel is a relevant marker of intestinal ischemia as it typically shows the effect of poor blood flow first, as it is the end area of intestinal perfusion.

Several techniques have been developed to quantitatively assess intestinal perfusion [3].

However, there is currently no widely accepted and/or readily available intraoperative tool to quantify intestinal blood flow or oxygenation, and therefore to assess viability. Reasons for low penetration of ancillary systems to guide intestinal resection in the operating room are manifold. Some systems are fairly expensive, or operator-dependent, and time-consuming.

A novel concept of fluorescence-based enhanced-reality (FLER) for real-time intraoperative assessment of intestinal perfusion has recently been proposed by our group [4]. FLER showed the ability to exactly detect the future resection line in a laparoscopic model of mesenteric ischemia.

Confocal laser endomicroscopy (CLE) is a high-resolution microscopic imaging modality with a selective depth of focus. Confocal images of intestinal mucosa can provide information on microcirculation based on capillary density or circulating blood cell velocity as well as on the morphology of intestinal villi and crypts demonstrating changes induced by the ischemic injury [5].

The aim of this study was to assess the ability of the Cellvizio $^{\circledR}$, a probe-based CLE system (Mauna Kea Technologies, France), to detect the "histological" modifications of the colonic mucosa induced by mesenteric ischemia in a porcine model. In addition, the study aims to assess the accuracy of the Cellvizio ${ }^{\circledR}$ in discriminating the transition zone from ischemic to vascularized areas, and to compare it to the FLER system, which evaluates colonic seromuscular changes.

\section{Materials and methods}

Animals

The present experimental study is part of a larger experimental protocol on intestinal ischemia (No. 38.2012.01.039), which received approval from the local
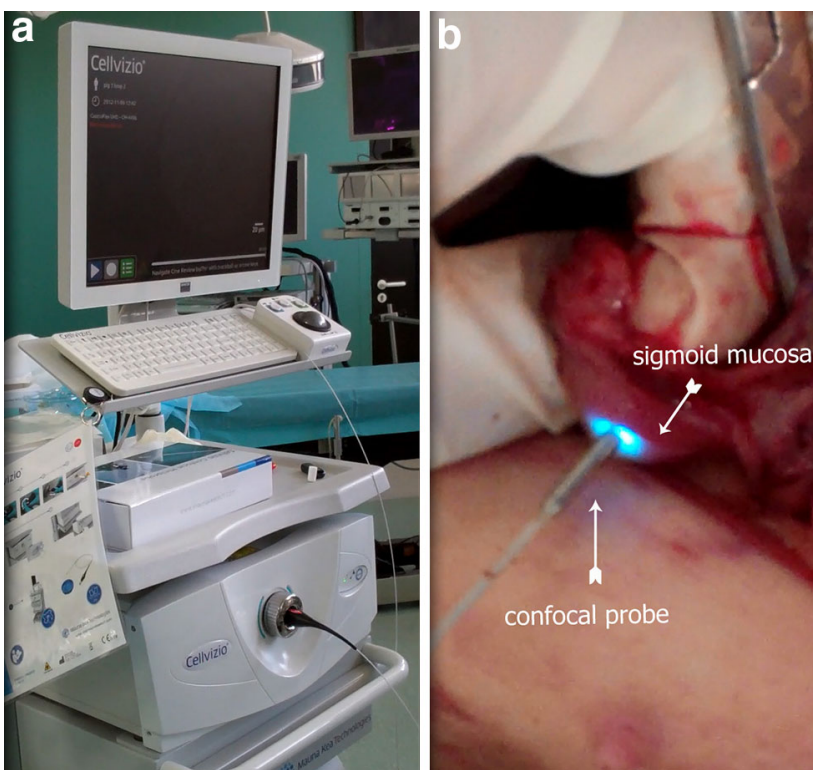

Fig. 1 Cellvizio ${ }^{\circledR}$ confocal probe applied directly to the sigmoid mucosa in an open model of sigmoid ischemia A Cellvizio ${ }^{\circledR}$ (Mauna Kea Technologies, France) probe-based confocal laser endomicroscopy system; B in the pilot study, confocal laser endomicroscopy of the sigmoid mucosa was performed by directly handling the probe and applying it on the mucosa through a full-thickness enterotomy

Ethical Committee on Animal Experimentation. All animals used in the experimental laboratory were managed according to French laws for animal use and care and according to the directives of the European Community Council (2010/63/ EU). Pigs were fasted for $24 \mathrm{~h}$ before surgery with free access to water. Premedication by intramuscular injection of ketamine $(20 \mathrm{mg} / \mathrm{kg})$ and azaperone $(2 \mathrm{mg} / \mathrm{kg})$ (Stressnil; Janssen-Cilag, Belgium) was administered $1 \mathrm{~h}$ before surgery. Induction was achieved by intravenous propofol $(3 \mathrm{mg} / \mathrm{kg})$ combined with pancuronium $(0.2 \mathrm{mg} / \mathrm{kg})$. Anesthesia was maintained with $2 \%$ isoflurane. A rectal enema was performed on the operative table until the effluent turned clear. At the end of the procedure, the pig was sacrificed according to our protocol with an intravenous injection of a lethal dose of potassium chloride.

A total of nine adult swine (Sus scrofa domesticus, ssp. Large White) were used in this non-survival study. Proof of the concept and technical clues to recognize intestinal ischemia using CLE were initially established in three pigs using an open surgery model of intestinal ischemia. Six animals [four males and two females, mean weight: $27.01 \mathrm{~kg}$ (SD 6.01)] were prospectively involved in the study protocol to assess the performance of the CLE probe in identifying viable/perfused resection margins when compared to clinical evaluation and FLER videography. 
Fig. 2 Fluorescence-based enhanced reality and confocal laser endomicroscopy analysis of resection lines in a laparoscopic model of sigmoid ischemia A Sigmoid loop suspended to the abdominal wall; B Blinded clinical evaluation of resection lines (green): the window in the mesentery was hidden by overlapping a white spot and surgeons drew two resection lines on a PC monitor. They placed two clips on the corresponding lines projected onto the operating monitor; $\mathbf{C}$ fluorescence signal as seen in near-infrared mode after injection of indocyanine green; D Virtual perfusion cartography obtained with image analyzer software (VR-RENDER ${ }^{\circledR}$ PERFUSION); E Enhanced reality obtained by overlapping virtual cartography with realtime laparoscopic images: resection lines $(2 \mathrm{a}=$ distal and $2 \mathrm{~b}=$ proximal) are outlined with a surgical marker; $\mathbf{F}$ the confocal probe analyzes the resection line $2 \mathrm{a}$ according to FLER (Color figure online)
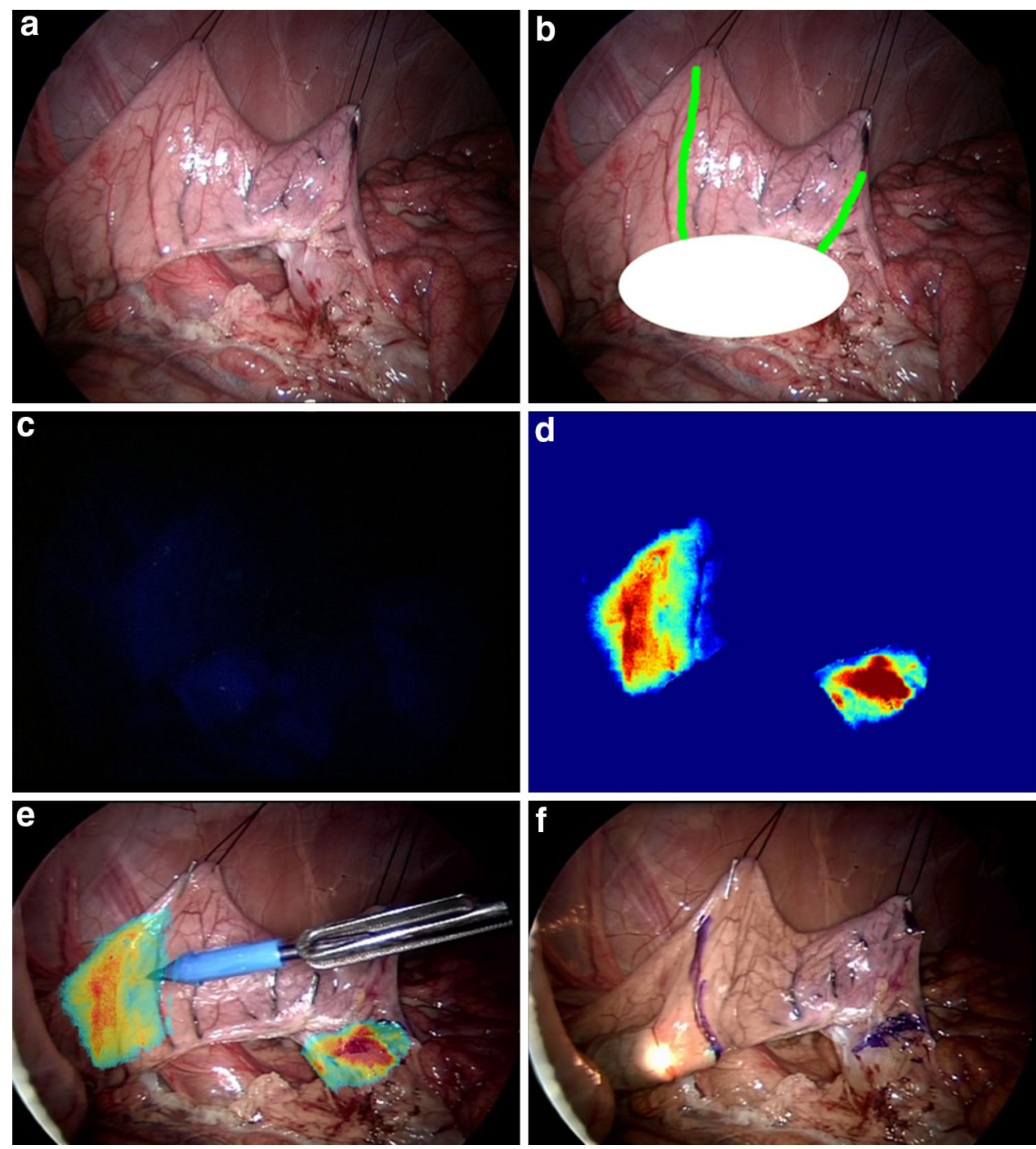

Procedures and equipment

\section{Open surgery model (pilot)}

In the pilot study, three pigs had a median laparotomy, and segmental $(4-5 \mathrm{~cm})$ ischemia of the sigmoid colon was induced by the application of vascular clamps on the terminal arterial branches. Resection margins were clinically evaluated at different time points (from 1 to $3 \mathrm{~h}$ ) after establishment of ischemia. Confocal laser endomicroscopy of the sigmoid mucosa was performed by directly handling the probe and applying it on the mucosa through a fullthickness enterotomy (Fig. 1).

\section{Laparoscopic surgery model}

Six pigs underwent a laparoscopic sigmoid segmental ischemia by sealing terminal arterial branches using the LigaSure $^{\mathrm{TM}}$ vessel-sealing device (Covidien, Boulder,
Colorado). The Inferior Mesenteric Artery (IMA) was spared. The sigmoid loop was suspended to the abdominal wall using transparietal sutures (Fig. 2A). Using different assessment modalities of bowel vascularization, five regions of interest (ROI) were defined as ischemic ROI 1, presumed viable margins (future resection lines) ROI $2 \mathrm{a}$ (distal to the ischemic zone) and 2b (proximal to the ischemic zone), and well-vascularized areas $3 \mathrm{a}$ (distal) and $3 \mathrm{~b}$ (proximal).

Clinical evaluation of the ischemic area One hour after interrupting the sigmoid vascular supply, the ischemic area was clinically evaluated to determine presumed viable colonic margins solely based on the aspect of the serosal surface by a second "blinded" surgeon. Blinding was obtained by superimposing a white spot onto the laparoscopic monitor, hence impeding the evaluating surgeon from estimating viable margins using apparent vessels as a landmark (Fig. 2B). Blinding on vascular tree distribution was deemed necessary in this experimental model since the 
porcine sigmoid mesentery is very thin and allows for a clear visualization of branch distribution to the sigmoid serosa, which is not the case in the majority of human cases. Presumed distal and proximal resection lines ( $2 \mathrm{a}_{\text {CLINIC }}$ and $2 \mathrm{~b}_{\text {CLINIC }}$ ) were marked with a surgical clip on the serosal site.

Fluorescence-based enhanced reality (FLER) Immediately after clinical evaluation, $0.5 \mathrm{mg} / \mathrm{kg}$ of Indocyanine Green (Infracyanine ${ }^{\circledR}$, Serb, Paris) was injected intravenously into the animal, and the D-Light-P (Karl Storz, Tuttlingen, Germany) was shifted to a near-infrared mode to capture the fluorescence signal (Fig. 2C). This fluorescence signal was analyzed using the Ad'Hoc software (ER PERFUSION, IRCAD) to construct virtual perfusion cartography of the sigmoid colon based on time-to-peak (Fig. 2D). Time-to-peak (in seconds) corresponds to the mean time elapsed for the fluorescence signal to reach its maximum intensity in a given area. This is averaged on the recorded video for at least $20 \mathrm{~s}$ at the speed of five frames per second. The software algorithm was set beforehand to observe an increase in time-to-peak of at least $50 \%$ when compared to a control area ( $3 \mathrm{a}$ or $3 \mathrm{~b}$ ). The slope of the increase is dependent upon perfusion. Virtual perfusion cartography generated by the software was overlapped onto the images shown on the laparoscopic monitor and subsequently superimposed onto the sigmoid colon, hence obtaining the Enhanced Reality effect (Fig. 2E). FLER and clinical evaluation for future resection lines $2 \mathrm{a}(n=6)$ and $2 \mathrm{~b}(n=6)$ were considered concordant when distance between the two landmarks (clip for clinic and surgical marker for FLER) on the sigmoid serosa was $<1 \mathrm{~cm}$. This definition of concordance comes from the width of laparoscopic linear staplers $(10 \mathrm{~mm})$ commercially available today. It would be the minimum discriminating distance for the application of a laparoscopic stapler.

Cellvizio ${ }^{\circledR}$ CLE In both open and laparoscopic models, pigs were injected intravenously with $2 \mathrm{~mL}$ of $10 \%$ fluorescein (Fluocyne ${ }^{\circledR}$, Serb, Paris, France). A $2.6-\mathrm{mm}$ confocal probe (Gastroflex ${ }^{\circledR}$ confocal Ultra High Definition Miniprobe; resolution $1 \mu \mathrm{m}$, field of view $240 \mu \mathrm{m}$, depth of focus $60 \mu \mathrm{m}$, and optical slice thickness $10 \mu \mathrm{m}$ ) was used. In the pilot study (open model), the probe was manipulated manually as it was being inserted in the operating channel of a standard colonoscope in the laparoscopic study. Immediately after injection of fluorescein, CLE probe scanning of the intestinal mucosa was performed following the lines drawn on the bowel wall according to FLER (Figs. 2F, 3, 4) and clinical evaluation (marked with clips) based on the serosal aspect. Digital videos of confocal scanning were recorded moving from the center of the ischemic ROI 1 (ROI 1 center) toward presumed viable margins and vascular areas first distally ( $2 a$ and $3 a$ ), and then proximally ( $2 b$ and $3 b$ ). The areas in which confocal imaging presented a sudden change were compared to those of FLER evaluation, which were previously marked with the surgical marker.

Conventional histology and ischemia score After confocal evaluation, a laparotomy was performed, and fullthickness surgical biopsies were taken at the ischemic area and at a control site (vascular areas $3 \mathrm{a}$ and $3 \mathrm{~b}$ alternately)
Fig. 3 Confocal probe scanning with real-time endomicroscopic imaging following fluorescence-based enhanced reality assessment Cellvizio ${ }^{\circledR}$ probe scanning of the intestinal mucosa was performed following the lines drawn on the intestinal wall according to FLER (marked with surgical marker) and clinical evaluation (marked with clips) based on the serosal aspect. Digital videos of confocal scanning were recorded moving from the center of the ischemic ROI 1 (ROI 1 center) toward presumed viable margins and vascular areas first distally (2A and $3 \mathrm{~A}$ ) and then proximally ( $2 \mathrm{~B}$ and 3B)

\section{ROI 1 ischemic}

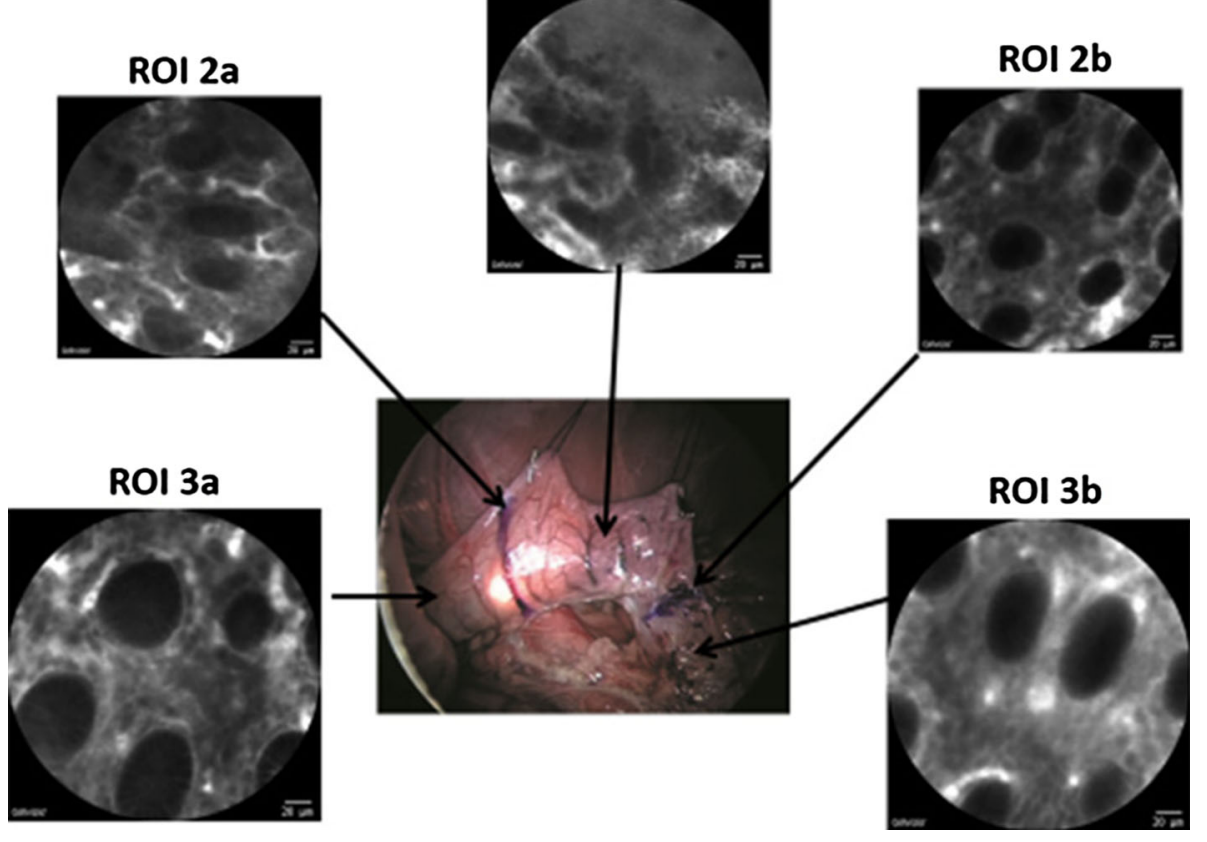


Fig. 4 Confocal laser endomicroscopy and fluorescence-based enhanced reality setting. The confocal probe is advanced into the sigmoid colon (A) following the drawings made on the serosa based on FLER (B). The confocal analysis $(\mathbf{C}$; arrow) shows "target cells" in correspondence to the periphery of the ischemic region. Detail of the endoscopic view showing the probe in contact with the mucosa $(\mathbf{D})$

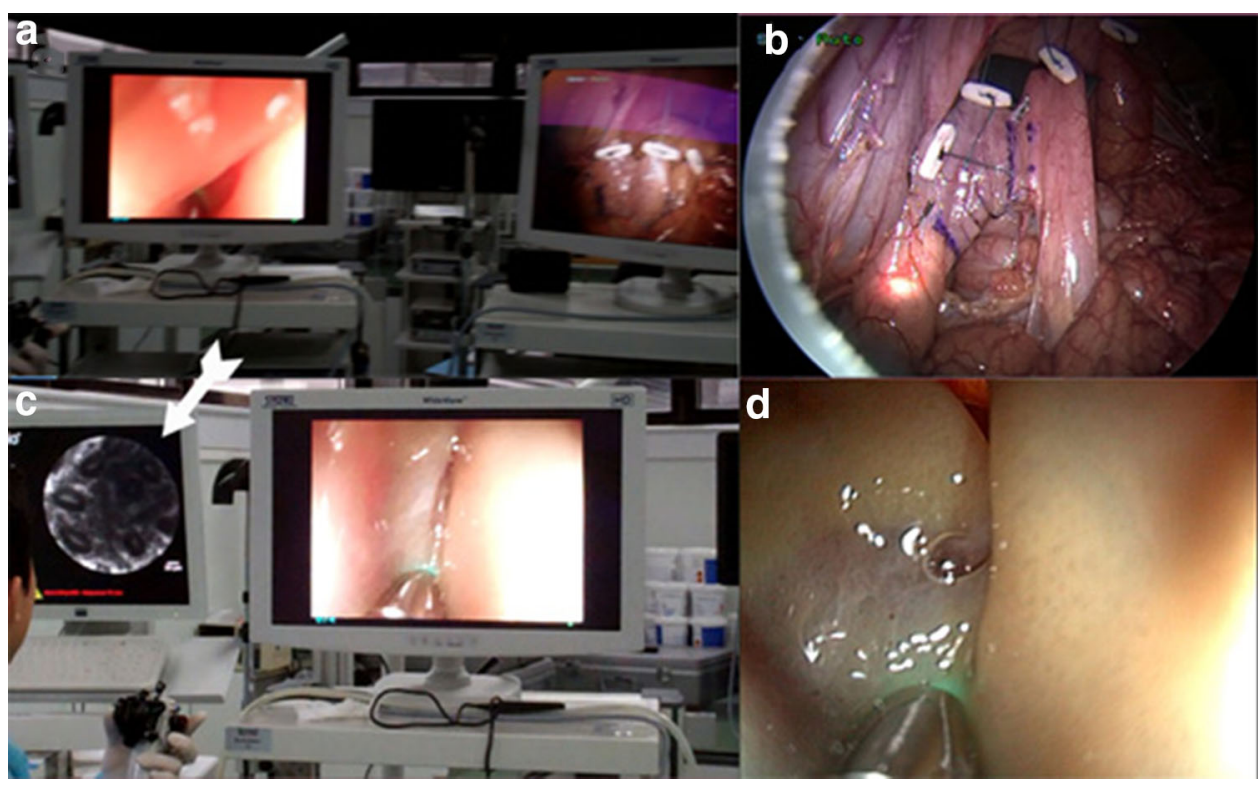

of the sigmoid colon. Specimens were fixed in $4 \%$ buffered formalin for at least $24 \mathrm{~h}$. Sections ( $4 \mu \mathrm{m}$ thick) were cut from paraffin-embedded tissues and stained with hematoxylin and eosin. Six sections per biopsy were analyzed. The same standardized semi-quantitative histology score to evaluate ischemia was applied by a blinded pathologist to conventional histology and confocal imaging. The score was made up as follows: $0=$ normal mucosa; 1 = partial epithelial edema and necrosis; $2=$ diffuse swelling and necrosis of epithelium; $3=$ necrosis with submucosal neutrophil infiltration; $4=$ widespread necrosis and massive neutrophil infiltration and bleeding. The pathologist was blinded to confocal digital video sequencing of ROIs.

Local capillary lactates Local capillary lactates were measured on blood samples obtained by puncturing the bowel serosa at ROIs as identified by FLER and by placing the sample on test strips connected to the $\mathrm{EDGE}^{\mathrm{TM}}$ Blood Lactate Analyzer (Apex Biotechnology Corp. Hsinchu, Taiwan, People's Republic of China). In case of discordance ( $>1 \mathrm{~cm}$ distance) between FLER and CLINIC at future resection lines, additional capillary lactates were measured at the sites identified by CLINIC. Systemic lactatemia was measured on blood samples obtained by puncturing the groin of the pig and by standard blood gas analysis performed on arterial blood obtained from the femoral artery.

\section{Statistical analysis}

Statistical analysis was performed using the GraphPad Prism Software. ANOVA followed by a Dunnet's multiple comparison test was performed to compare the ischemic zone to future resection lines and vascularized areas. A Student's $t$ test was used to calculate $\mathrm{p}$ values for continuous variables. A $p$ value $<0.05$ was considered statistically significant.

\section{Results}

(1) Fluorescence-based enhanced reality and clinical evaluation Clinical $\left(2 \mathrm{a}_{\text {CLIN }}-2 \mathrm{~b}_{\text {CLIN }} ; n=12\right)$ and FLER $\left(2 \mathrm{a}_{\mathrm{FLER}}-2 \mathrm{~b}_{\mathrm{FLER}} ; n=12\right)$ regions of interests (ROI) were compared. In $50 \%$ of cases $(n=6)$, there was a concordance $(<1 \mathrm{~cm}$ distance) between clinical assessment and FLER imaging for resection lines ( $2 a$ and $2 b$ ). In five cases, resection lines according to clinical assessment were closer to the ischemic zone with a mean distance of $2.2 \mathrm{~cm}$ (SD 1.78) when compared to FLER assessment.

(2) Capillary lactates The one-way ANOVA followed by Dunnett's multiple comparison test showed that mean capillary lactate levels were significantly higher at the ischemic area (ROI 1; $4.91 \mathrm{mmol} / \mathrm{L}$; SD 1.87; $95 \%$ confidence interval (CI) 2.95-6.88) than in the presumed resection margins as assessed by FLER $\left(2 \mathrm{a}_{\mathrm{FLER}}-2 \mathrm{~b}_{\mathrm{FLER}}\right.$; mean $2.8 \mathrm{mmol} / \mathrm{L}$; SD 1.69 ; mean difference $2.11 ;$ q $2.795 ; 95 \%$ CI $0.26-3.97$; $p<0.05)$ and to vascular areas $(3 a-3 b)$ in which values were $1.9 \mathrm{mmol} / \mathrm{L}$ (SD 0.48 ; mean difference 3.01; q 3.98; $95 \%$ CI 1.163-4.871; $p<0.001$ ). There was no statistically significant difference in local lactatemia between the ischemic zone and presumed viable margins as assessed by clinical evaluation $\left(2 a_{\text {CLINIC }}-2 b_{\text {CLINIC }}\right)$. Taking into account discordant 
Fig. 5 Summary of confocal assessment of ischemic and non-ischemic areas. At the center of the ischemic area, an almost constant finding was the presence of "blurred images" due to the absence of penetration of fluorescein into the ischemic tissue. Still within the ischemic zone, but closer to resection lines, a consistent change was the presence of "target cells", with a clear center probably induced by some fibrin deposits in the glandular lumen of enterocytes. This "target cell" image was not visible in vascular zones, in which the center of enterocytes was constantly black

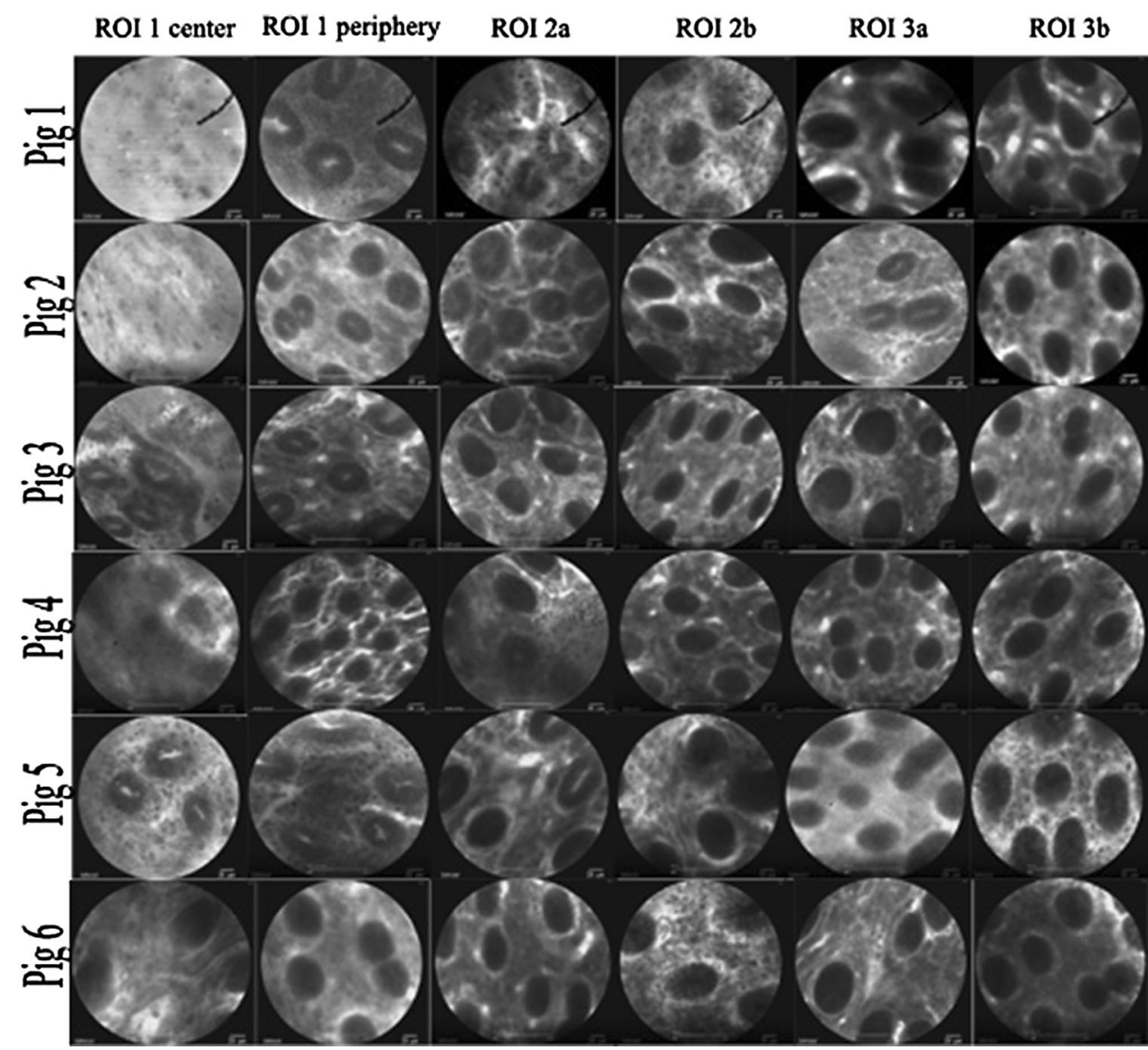

cases $(n=6)$ separately, mean capillary lactates were significantly higher at $2 \mathrm{a}_{\text {CLINIC }}+2 \mathrm{~b}_{\text {CLINIC }}$ when compared to $2 \mathrm{a}_{\mathrm{FLER}}+2 \mathrm{~b}_{\mathrm{FLER}} \quad(3.24 \pm 0.87$ vs. $1.94 \pm 0.45 \mathrm{mmol} / \mathrm{L} ; p<0.05)$. In one case, FLER was closer to the ischemic area with higher lactate values $(3.7 \mathrm{mmol} / \mathrm{L})$ when compared to clinical assessment $(1.7 \mathrm{mmol} / \mathrm{L})$. There was no statistically significant difference between vascular areas and presumed viable margins. Mean arterial lactatemia assessed by standard blood gas analysis was $1.385 \mathrm{mmol} / \mathrm{L}$ (SD 0.24). It was statistically significantly lower when compared to capillary systemic lactatemia assessed with the $\mathrm{EDGE}^{\mathrm{TM}}$ analyzer $(1.76 \mathrm{mmol} / \mathrm{L}$ (SD 0.287); $p=0.006)$. Both capillary and arterial systemic lactatemia were significantly lower when compared to capillary lactatemia at the ischemic ROI 1 ( $p<0.002$ for both) but not significantly different from capillary lactatemia of the bowel measured at vascular areas $(p=0.23$ and $p=0.27$, respectively).

(3) Confocal assessment The almost constant endomicroscopic changes reflecting the presence of hypoperfusion could be identified (1) at the center of ROI $l$ as a blurred image with inability to obtain clear "confocal" imaging of enterocytes, and (2) at the periphery of ROI 1 (still within the identified ischemic area but closer to one of the future resection lines), as the presence of a "target cell" image of enterocytes. A sudden change when reaching vascular areas with clear imaging of enterocytes was observed when the areas were scanned by the probe corresponding to the ones identified by $2 a_{F L E R}-2 b_{F L E R}$ in $11 / 12$ cases versus $7 / 12$ in $2 a_{\text {CLINIC }}-2 b_{\text {CLINIC }}$ (six cases concordant with FLER and one discordant). In one case (pig 6), there were no target cells (Fig. 5). In addition to specific features caused by fluorescein injection, it was possible to apply the same score used in conventional pathology demonstrating the presence of edema, disruption of epithelium, and necrosis with submucosal neutrophil infiltration (Table 1).

(4) Comparison between confocal and standard pathology using a standardized score. In the pilot phase, the ischemia score confocal analysis ranged from 0 (normal mucosa) after 1-h ischemia, increasing to 2 (diffuse swelling and necrosis of epithelium) after $3 \mathrm{~h}$. In the laparoscopic 1-h ischemia model, mean ischemia score at the ROI 1 ischemic zone was 
Table 1 Comparison between standard and confocal microscopy evaluation of 1-h sigmoid ischemia using a standardized score

\begin{tabular}{|c|c|c|c|c|c|c|c|c|c|c|}
\hline \multirow[t]{2}{*}{ PIG N } & \multicolumn{3}{|c|}{$\begin{array}{l}\text { Standard } \\
\text { pathology } \\
\text { score (ROI) }\end{array}$} & \multicolumn{5}{|c|}{$\begin{array}{l}\text { Confocal score } \\
\text { (ROI) }\end{array}$} & \multirow[t]{2}{*}{$\begin{array}{l}\text { Comments standard } \\
\text { pathology ROI } 1\end{array}$} & \multirow[t]{2}{*}{ Comments confocal } \\
\hline & 1 & $3 a$ & $3 b$ & 1 & $2 \mathrm{a}$ & $2 b$ & $3 a$ & $3 \mathrm{~b}$ & & \\
\hline 1 & 1 & 0 & - & 3 & 1 & 0 & 0 & 0 & $\begin{array}{l}\text { Focal epithelial erosion and } \\
\text { submucosal congestion (ROI 1) }\end{array}$ & $\begin{array}{l}\text { Absence of enterocytes silhouette. } \\
\text { Target cells image in ROI } 1 \text { and 2a. }\end{array}$ \\
\hline 2 & 1 & - & 0 & 2 & 0 & 0 & 0 & 0 & & Congestion, target cells images in ROI $1,2 \mathrm{a}$ and $3 \mathrm{a}$ \\
\hline 3 & 1 & 0 & - & 2 & 1 & 0 & 0 & 0 & & Congestion, target cells images in ROI 1 \\
\hline 4 & $0 / 1$ & - & 0 & 1 & 0 & 0 & 0 & 0 & $\begin{array}{l}\text { Minimal inflammatory reaction } \\
\text { and submucosal congestion }\end{array}$ & $\begin{array}{l}\text { Blurred image at the center but normal } \\
\text { mucosa at the periphery of ROI } 1 .\end{array}$ \\
\hline 5 & 1 & 0 & - & 1 & 1 & 0 & 0 & 1 & $\begin{array}{l}\text { Focal epithelial erosion and submucosal } \\
\text { congestion (ROI 1) }\end{array}$ & $\begin{array}{l}\text { Congestion in ROI } 1 \text { and } 3 \mathrm{~b}, \\
\text { target cells in ROI } 1 \text { and } 2 \mathrm{a}\end{array}$ \\
\hline 6 & 0 & - & 0 & 1 & 0 & 1 & 0 & 1 & Submucosal congestion & Congestion in ROI 1 and $2 b$ \\
\hline
\end{tabular}

statistically significantly higher at confocal assessment when compared to standard pathology 1.33 (SD 0.51) versus 0.66 (SD 0.51) $(p=0.01)$. At vascular areas, the score was invariably 0 (normal mucosa; $n=6$ ) at the standard pathology and was 0 in $10 / 12$ cases according to confocal analysis (Table 1). The overall Spearman correlation coefficient between standard pathology and confocal assessment was 0.685 , but it decreased to 0.5 when considering ischemic zones separately.

\section{Discussion}

Near-infrared fluorescence videography, is a promising approach for the intraoperative assessment of intestinal blood supply since it is easy to use, rapid, safe, and it does not imbalance the surgical workflow.

Carus and Dammer [6] proposed the use of laparoscopic fluorescence angiography in a series of 45 patients undergoing colorectal resections. To determine perfusion adequacy, Carus and Dammer used the degree of "relative blueness", as provided by computer analysis. However, this analysis is "static" and does not take into account the over-time diffusion of the fluorescent dye, which can reach ischemic areas by retrograde flow and provide incorrect information on the exact perfusion value. Additionally, fluorescent intensity could not be used for comparisons between patients without the use of a "standard" reference calibration tool (which is a squared spot that elicits a constant signal when illuminated by the near-infrared light that should be introduced into the abdomen of the patient).

Our group developed a dedicated image analyzer software (ER-PERFUSION, IRCAD, France) to obtain FLER [4]. FLER is performed using a near-infrared endoscope (D-Light P, Karl Storz, Tuttlingen, Germany), and the ER
PERFUSION software that generates a virtual perfusion cartography based on fluorescence time-to-peak. To assess time-to-peak, dye injection is performed in real time while looking at the region of interest, in order to capture and record the "dynamics" of the diffusion as well as the evolution of fluorescence. The virtual perfusion cartography is created by averaging the signals over a 20 - to $40-\mathrm{s}$ video at the speed of 5 frames per second and attributing a color code based on the time required to reach the maximum intensity for each pixel. Each single pixel making up virtual perfusion cartography is a dynamic image (2D + diffusion time) standing for the average of several images (from 100 to 200). Virtual cartography is overlapped with real-time laparoscopic images in order to obtain an "augmented reality" effect. The use of the fluorescence time-to-peak slope has two advantages when compared to the use of the absolute value of "fluorescence intensity". First, time-to-peak is independent of the distance between the light source and the imaged area. This is not the case with fluorescence intensity, which is highly dependent upon distance (a low perfused area may look more intensively fluorescent when observed closely and vice versa; a highly perfused area may look poorly fluorescent when observed from remotely). Secondly, time-topeak allows for multiple and repetitive assessments since the "noise" produced by the accumulation of fluorescent dye does not affect the steepness of the slope.

In the present study, a model of sigmoid ischemia was preferred over the small bowel to allow access with the confocal probe through a conventional endoscope and to explore microscopic ischemic changes of the colonic mucosa. In addition, it allows to determine their correlation to "external" serosa bowel signs detected by FLER. A 1-h ischemia length was chosen to be consistent with "ischemic time" occurring during a standard laparoscopic sigmoid resection with a vascular-first, medial-to-lateral approach. 
Similarly to the previous study on small bowel ischemia, FLER could identify the ischemic area (ROI 1) of the sigmoid colon and resection lines $(2 \mathrm{a}=$ distal and $2 \mathrm{~b}=$ proximal). FLER was compared to the clinical evaluation made by two blinded surgeons (BD and $\mathrm{YN}$ who are consultant and PGY8, respectively). In $50 \%$ of cases, distance measured at the laparotomy site between clips (clinical estimation) and the drawing made with the surgical marker under FLER guidance was less than $1 \mathrm{~cm}$, and, as a result, evaluations were defined as concordant. However, in all but one case, the FLER-guided marking was invariably more distant to the ischemic zone.

Lactate levels reflect cellular suffering caused by hypoxic conditions with a shift toward anaerobic energetic metabolism. Systemic lactate increase is slow, useless for early diagnosis of mesenteric ischemia, and has mainly a prognostic value [7]. Local lactates increase significantly faster and might be a useful intraoperative guide to select the most adapted resection site, especially in early mesenteric ischemia when the serosal color is insufficiently informative regarding bowel perfusion and viability. In this study, capillary lactate levels were significantly higher at the ischemic zone when compared to resection lines as assessed by FLER but there was no difference between the ischemic area and resection lines assessed by clinical evaluation. The threshold value of capillary lactates ensuring optimal anastomotic healing is currently under evaluation at our research institute in a survival experimental model.

It is not currently known how ischemic changes of colonic intestinal mucosa correlate to changes in seromuscular layers, or whether one is a better predicting factor of anastomotic ischemia as compared to the other. The main objective of this study was to determine if there is indeed any measurable difference between mucosal ischemic changes as evidenced by CLE and visually defined or FLER-determined ischemia.

Cellvizio $^{\circledR}$ (Mauna Kea Technologies, Paris, France) is a probe-based CLE system. The probe can be inserted through conventional endoscopes, and allows for intraoperative microscopic imaging of neoplastic and inflammatory lesions virtually throughout the entire gastrointestinal tract [8]. The clinical interest of confocal technology in the evaluation of neoplasia in Barrett's metaplasia [9], gastric [10], and colorectal [11] neoplasia seems to be increasingly confirmed.

In this paper, we aimed to assess the ability of CLE to evaluate intestinal mucosa changes at the future anastomotic site and to correlate real-time histology to the more clinically adapted FLER technique.

Cellvizio $^{\circledR}$ is not meant for the evaluation of intestinal ischemia, and as a result, no reference atlas on mucosal changes due to ischemia is available. In the pilot study, in which multiple assessments at different ischemia times were made to evaluate the technique's feasibility, some obvious changes were observed as early as $1 \mathrm{~h}$ after ischemia induction, and in the prospective study, all animals underwent 1-h ischemia. The inability to obtain a clear confocal image of enterocytes and crypts was an almost constant finding at the center of the ischemic area due to the absence of penetration of fluorescein into the ischemic tissue. Moving toward future resection lines but still within the ischemic zone, a consistent change was the presence of enterocytes with a "target cell" image, with a clear acinar center. This was due to a distortion of the glandular lumen, with increased permeability to fluorescein. This "target cell" image was not visible in vascular zones, in which the center of enterocytes was constantly black (Fig. 5).

Yasumura et al. [12] used a charge-coupled microscopic device to perform the microscopic analysis of the serosal bowel layer. It could calculate the ratio of blood cells to the effective intestinal vascular bed as a predictive index of bowel survival. This technique which quantifies blood flow objectively seems to be very promising and useful, unless it is time-consuming.

However, CLE requires the mobilization of too many resources. It is time-consuming and less adapted than FLER to routine use in clinical practice for anastomotic perfusion assessment. Nonetheless, CLE remains a very appealing technology for some clinical applications such as stoma perfusion assessment. Additionally, the ability to visually follow up intestinal perfusion could be of paramount importance, especially in the Intensive Care Unit, to adjust resuscitation treatment in patients undergoing an open abdomen procedure or in patients treated for abdominal compartment syndrome. This might have a prognostic value in septic shock of abdominal origins. Schmidt et al. [13] used CLE to detect sepsis-related changes in intestinal mucosal microcirculation in a porcine model of septic shock and also in patients in severe sepsis and in healthy controls.

It was uneasy to correlate confocal ischemia score to standard pathology score. Estimation of confocal videos seemed to overestimate ischemic changes when compared to standard pathology. Reference confocal images were obtained in the pilot phase in three pigs in which multiple analyses were performed including a longer ischemia time. Changes noted in the prospective study at the conventional HE staining were invariably mild, and this might have affected the interpretation although reasonably standardized. The model of ischemia was strictly similar in both phases: the inferior mesenteric artery was spared and only terminal branches were sealed using the LigaSure ${ }^{\mathrm{TM}}$ vessel-sealing device (Covidien, Boulder, Colorado). This could well account for low ischemia scores. However, it has to be stressed that a fine microscopic assessment does not have a real-time surgical use, and consequently the comparison was beyond the scope of the study. However, it 
Fig. 6 Comparative images of standard histology and confocal analysis matched according to the ischemia score assigned by a blinded pathologist Matched images at standard pathology and confocal analysis. $\mathbf{A} \times 20$ Hematoxylin-eosin (HE) and B confocal imaging score 0 (normal mucosa); $\mathbf{C} \times 20 \mathrm{HE}$ and $\mathbf{D}$ confocal imaging score 1 (submucosal congestion and swelling); $\mathbf{E} \times 40 \mathrm{HE}$; and F confocal imaging score 2 (diffuse swelling and necrosis of epithelium)
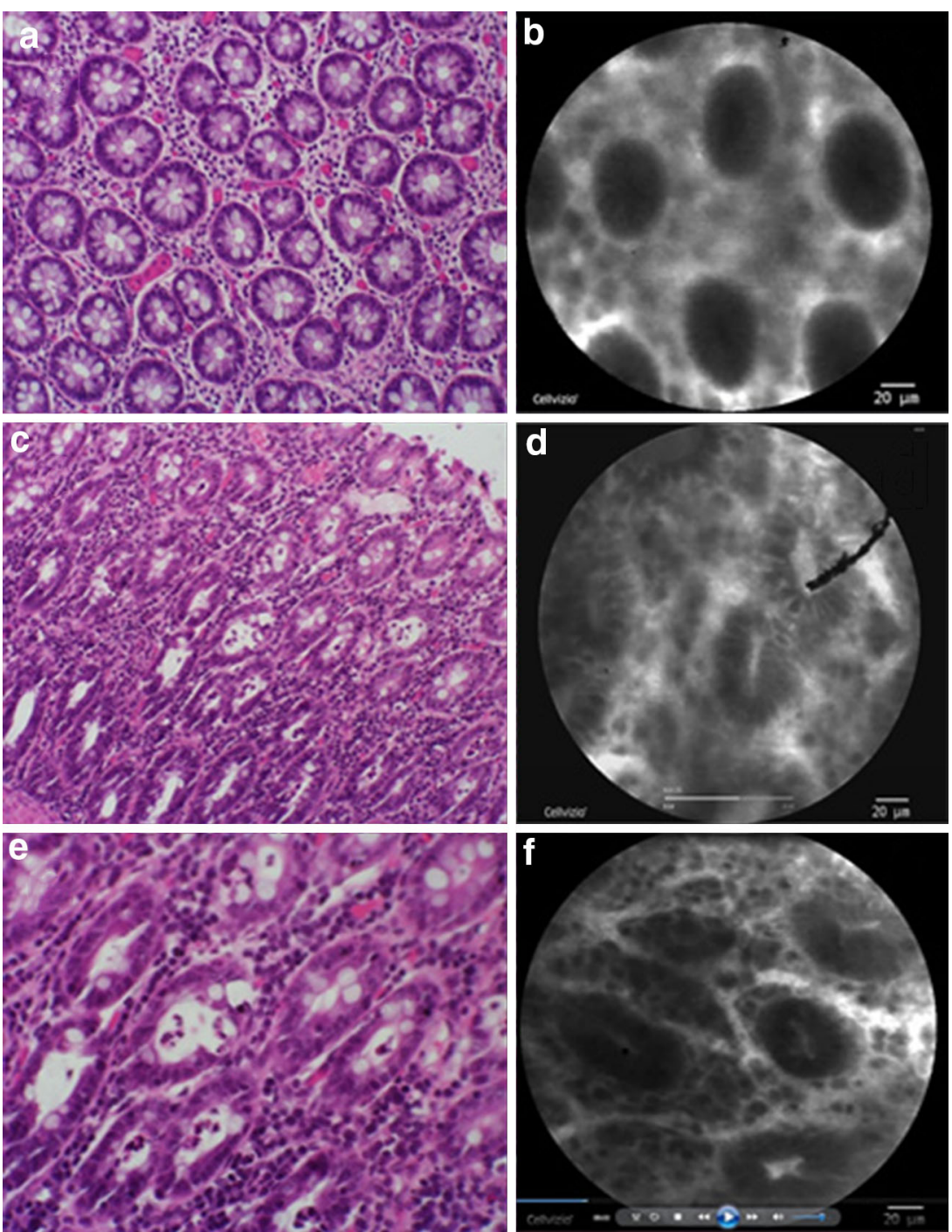

was possible to match some confocal analyses with corresponding $\mathrm{HE}$ images (Fig. 6). The dynamic aspect of confocal scanning provides the ability to identify precise clues at any level of ischemia and yields advantages regarding the static picture of standard pathology. It also allows for more precise assessments.

The primary weakness of this non-survival study was the lack of correlation of relative ischemic zones identified by either test to clinically significant anastomotic ischemia. The next obvious step will be survival studies with resections performed in the various zones of imaged ischemia to control how they heal.

\section{Conclusions}

FLER was more accurate than clinical evaluation to determine future resection lines as expressed by the level of capillary lactates. Confocal analysis confirmed that FLER is effective to discriminate the transition zone at the future anastomotic site. It showed endomicroscopic features specific to ischemic and vascularized areas of the bowel after 1-h ischemia. Although such findings require confirmation in the clinical setting, probe-based confocal analysis might represent a potential tool for "micro-image-guided intestinal resections".

Acknowledgments This study was partly funded by a research grant from Karl Storz, Tuttlingen, Germany. The authors are grateful to Christopher Burel and Guy Temporal (medical English reviewers working for IRCAD-IHU Institutes) for their valuable help in proofreading the manuscript.

Disclosure Michele Diana is recipient of a research grant from Karl Storz, Tuttlingen, Germany. Karl Storz was NOT involved in study design nor data acquisition/interpretation. Jacques Marescaux is the President of the IRCAD-IHU Institutes, partly funded by Karl Storz Endoscopy, Covidien, and Siemens Healthcare. Remaining authors have no conflicts of interest or financial ties to disclose. 


\section{References}

1. Karliczek A, Benaron DA, Baas PC et al (2010) Intraoperative assessment of microperfusion with visible light spectroscopy for prediction of anastomotic leakage in colorectal anastomoses. Colorectal Dis 12:1018-1025

2. Karliczek A, Harlaar NJ, Zeebregts CJ et al (2009) Surgeons lack predictive accuracy for anastomotic leakage in gastrointestinal surgery. Int J Colorectal Dis 24:569-576

3. Urbanavicius L, Pattyn P, de Putte DV et al (2011) How to assess intestinal viability during surgery: a review of techniques. World J Gastrointest Surg 3:59-69

4. Diana M, Noll E, Diemunsch P et al (2014) Enhanced-reality video fluorescence: a real-time assessment of intestinal viability. Ann Surg 259(4):700-707

5. Laemmel E, Genet M, Le Goualher G et al (2004) Fibered confocal fluorescence microscopy (Cell-viZio) facilitates extended imaging in the field of microcirculation. A comparison with intravital microscopy. J Vasc Res 41:400-411

6. Carus T, Dammer R (2012) Laparoscopic fluorescence angiography with indocyanine green to control the perfusion of gastrointestinal anastomoses intraoperatively. Surg Technol Int 22:27-32

7. Alves WF, Aguiar EE, Guimaraes SB et al (2010) L-alanyl-glutamine preoperative infusion in patients with critical limb ischemia subjected to distal revascularization reduces tissue damage and protects from oxidative stress. Ann Vasc Surg $24: 461-467$

8. Kuiper T, van den Broek FJ, van Eeden S et al (2011) New classification for probe-based confocal laser endomicroscopy in the colon. Endoscopy 43:1076-1081

9. Wu J, Pan YM, Wang TT et al (2014) Confocal laser endomicroscopy for detection of neoplasia in Barrett's esophagus: a meta-analysis. Dis Esophagus 27(3):248-254

10. Wang SF, Yang YS, Wei LX et al (2012) Diagnosis of gastric intraepithelial neoplasia by narrow-band imaging and confocal laser endomicroscopy. World J Gastroenterol 18:4771-4780

11. Dong YY, Li YQ, Yu YB et al (2013) Meta-analysis of confocal laser endomicroscopy for the detection of colorectal neoplasia. Colorectal Dis 15:e488-e495

12. Yasumura M, Mori Y, Takagi H et al (2003) Experimental model to estimate intestinal viability using charge-coupled device microscopy. Br J Surg 90:460-465

13. Schmidt C, Lautenschlager C, Petzold B et al (2013) Confocal laser endomicroscopy reliably detects sepsis-related and treatment-associated changes in intestinal mucosal microcirculation. Br J Anaesth 111(6):996-1003 